\title{
The Influence of Deconvolution on Surface-Related Multiple Elimination Filtering
}

\author{
Laila D. L. Vergne, CPGG/UFBA, Milton J. Porsani, CPGG/UFBA
}

Copyright 2019, SBGf - Sociedade Brasileira de Geofísica.

This paper was prepared for presentation at the $16^{\text {th }}$ International Congress of the Brazilian Geophysical Society, held in Rio de Janeiro, Brazil, 19 August to 22 August $2019,2019$.

Contents of this paper were reviewed by the Technical Committee of the $16^{\text {th }}$ International Congress of The Brazilian Geophysical Society and do not necessarily represent any position of the SBGf, its officers or members. Electronic reproduction or storage of any part of this paper for commercial purposes without the written consent of The Brazilian Geophysical Society is prohibited.

\section{Abstract}

Using the methods of deconvolution it was possible to reach the objective of delimiting the reflections obtained by the seismogram, improving the temporal resolution. The following deconvolutions were used: Wiener deconvolution (spike), adaptive deconvolution, and $L_{p}$ norm deconvolution. Among the deconvolutions used, it was established which one showed the best result after application of the SRME filtering method in the seismic flow, where the effects of the surface multiple should be smoothed in the marine data of the Jequitinhonha Basin, making it possible to obtain a better seismic section closer to the subsurface geological reality of the study area.

\section{Introduction}

The deconvolution is a stage of seismic processing that seeks to increase the resolution of the data, so that the reflections become more visible. To understand this process it is necessary first to examine the constituent elements of a trace present in the seismogram. The earth is composed of layers of rock with different lithologies and physical properties. These layers are defined by their seismic impedance, in other words, by the product of the density of the layer with the velocity of propagation of the waves in the layer. The contrast of the impedances between adjacent layers causes the reflections that are recorded along the surface profile.

The model of a seismogram is given by the convolution between the impulse response of the earth and the seismic pulse. The earth impulse response, or reflectivity function, is what would be recorded if the pulse were just a unitary pulse. The impulse response comprises primary reflections and all possible multiple. An ideal deconvolution should compress the components of the pulse, leaving only the reflectivity of the earth in the seismic trace.

The pulse compression can be done by using a inverse filter as a deconvolution operator. A inverse filter when convoluted with the seismic pulse converts it into a unitary pulse. When applied to a seismogram, the inverse filter must provide the reflectivity function.

The work of Lima and Porsani (2013) discusses the conventional Wiener filtering or spike deconvolution, where the pulse must be of minimum phase and the reflectivity must be random, thus it is possible to obtain a causal inverse filter and the function of autocorrelation can be obtained directly of the seismic trace. The resolution of the output of the Wiener filtering can be controlled by designating a Wiener prediction error filter. Converting the seismic pulse into a unitary pulse is asking for perfect resolution. In practice, due to the noise in the seismogram and the assumptions made about the seismic pulse and the recording seismogram, spike deconvolution is not always desirable.

The deconvolution using the norm $L_{p}$ was used to develop the work of Lima and Porsani (2013) and Melo and Porsani (2001). It is able to perform the compression of the pulse using causal and non-causal filters, that is, by using this type of deconvolution it is possible to circumvent the premise of the minimum phase of the pulse. Thus, it is possible to apply the deconvolution with norm $L_{p}$ also for mixed phase pulses, and as a consequence these present better results than the conventional spike deconvolution.

Another premise of the conventional Wiener deconvolution is that the seismic pulse must be stationary along the seismic trace, which does not happen in reality. To solve this problem the adaptive deconvolution is used as an example in the work of Montenegro and Porsani (1995), and a different Wiener filter is applied for each portion of the seismic trace, which can be considered approximately stationary.

After obtaining all the deconvolved data, we will use these as input data for a processing flow where the multiple attenuation method used will be the SRME that predicts and subtracts the multiple from the original data.

It is important to note that the data processing of the Jequitinhonha Basin was carried out until the final stacking phase. In this way it was possible to visualize the data with the best resolution obtained. Part of the development of this work is done in ProMax/Seisspace processing software, and part is performed using the FORTRAN.90 language.

\section{Theory}

The seismic trace is the result of the interaction of the seismic pulse with the geological environment, from the moment the pulse is generated until the moment the reflections are recorded. In an analytical way the traces are the result of convolution of the seismic pulse generated in the seismic source with a series of reflection coefficients, the reflectivity function plus a linear operator representing the undesirable noise. The convolutional model is described mathematically by:

$$
x_{t}=p_{t} * e_{t}+n_{t},
$$

where:

$x_{t}=$ seismic trace;

$p_{t}=$ seismic pulse;

$e_{t}=$ reflectivity function;

$n_{t}=$ noise;

$*=$ convolution representation. 
Some hypotheses used for the construction of the seismic trace are:

- The earth consists of plane-horizontal layers with constant velocity;

- The source generates a compressional plane wave that reaches the layers in normal incidence. Under such circumstances the shear waves do not occur;

- The shape of the wave does not change in subsurface, it is stationary, it does not consider the amplitude decay (due to the divergence of the wave front) and the attenuation of the high frequencies (effects of absorption in the rocks);

- The noise component $n_{t}$ is negligible;

- The waveform of the source is known.

The deconvolution of a seismic pulse is convolution of the pulse with its inverse filter. If $\tilde{h}_{t}$ is an inverse approximation of the pulse $p_{t}$, then:

$$
\tilde{h}_{t} * p_{t} \approx \delta_{t}= \begin{cases}0 & \text { se } t \neq 0 \\ 1 & \text { se } t=0\end{cases}
$$

When the source waveform (source signature) is known, the problem is a deterministic one. This solution is given by the inverse filter. When this form is unknown, the problem is calculated statistically. There exists then a filter $\tilde{g}_{t}$ that convolved with the seismic trace $x_{t}$ will give the required function. This filter is called Wiener's prediction error operator.

\section{Wiener Filter}

In the deconvolution process some assumptions are taken into account: the pulse is of minimum phase, the reflectivity is white (Yilmaz, 1987), that is, random, and the pulse must be stationary along the seismic trace.

The Wiener-Levinson deconvolution has three stages:

1. It is estimated the coefficients of the autocorrelation function;

2. The inverse Wiener-Levinson filter is obtained;

3. The filter is applied to the seismic traces, through deconvolution.

The Wiener filter is causal $t=(0, \ldots, N)$. The autocorrelation function used is a challenge for the deconvolution, since, because it does not know the seismic pulse of the source, it is estimated directly from the seismic trace.

The seismic trace $x_{t}$ can be represented by the autoregressive model:

$$
\tilde{x}_{t}=\tilde{a}_{1} x_{t-1}+\ldots+\tilde{a}_{N} x_{t-N}
$$

The prediction error $e_{t}=x_{t}-\tilde{x}_{t}$ can be represented by:

$$
e_{t}=x_{t}-\tilde{a}_{1} x_{t-1}-\tilde{a}_{2} x_{t-2}-\ldots-\tilde{a}_{N} x_{t-N},
$$

or:

$$
e_{t}=\left[\begin{array}{llll}
x_{t} & x_{t-1} & \ldots & x_{t-N}
\end{array}\right]\left[\begin{array}{c}
1 \\
a_{1} \\
\vdots \\
a_{N}
\end{array}\right]
$$

where $a_{N}=-\tilde{a}_{N}$. The vector $\left(\begin{array}{llll}1 & a_{2} & \ldots & a_{n}\end{array}\right)$ represents the predictive operator of error and the coefficients $a_{N}$ se $N=$ $1,2,3$ are obtained with the method of the least squares, solving the system of normal equations below:

$$
\left[\begin{array}{llll}
r_{0} & r_{1} & r_{2} & r_{3} \\
r_{1} & r_{0} & r_{1} & r_{2} \\
r_{2} & r_{1} & r_{0} & r_{1} \\
r_{3} & r_{2} & r_{1} & r_{0}
\end{array}\right]\left[\begin{array}{c}
1 \\
a_{1} \\
a_{2} \\
a_{3}
\end{array}\right]=\left[\begin{array}{c}
E_{a 3} \\
0 \\
0 \\
0
\end{array}\right]
$$

$E_{a 3}$ is the sum total of the minimized square errors and $r_{N}$ represents the coefficients of the autocorrelation function of the seismic trace $x_{t}$. The matrix of normal equations is the Toeplitz band-structured autocorrelation matrix. This is a particular case of the least squares method and can be solved by Levinson's recursion.

\section{Deconvolution with Norm $L_{p}$}

The least squares method involves the observed values and the desired model present in the deconvolution that are found in the seismic trace and in the reflectivity, respectively. This method conventionally performs adjustment of the parameters by minimizing the norm $L_{2}$, that is, the sum of the squares of the deviations. Large deviations can be minimized with the norm $L_{1}$, that is, the sum of the absolute values of the deviations. The linearized inversion problem can be solved by establishing a linear approximation for the equation describing a given model. The norm definition of a vector extends the formalism of the linearized inversion to the norm $L_{p}$.

The great peculiarity of the deconvolution with norm $L_{p}$, is that it operates with both the causal and the anti-causal filters. This feature enables mixed-phase deconvolution. According to Porsani et al. (2001), Melo and Porsani (2001) and Popini et al. (2001) the objective function in terms of norm $L_{p}$ is written as follows:

$$
Q\left(\mathbf{a}^{+}, p\right)=\sum_{t=0}^{M+N}\left\{\left[\left(e_{t}\right)^{2}\right]^{1 / 2}\right\}^{p},
$$

where: $\mathbf{a}^{+}$is the vector with the elements of the causal filter, $p$ corresponds to the norm, $M$ the number of samples, $N$ the number of filter coefficients and $e_{t}$ represents the signal prediction error $x_{t}$, from the linear combination of their values to times past.

\section{Adaptive Deconvolution}

The method of deconvolution is not always satisfactory, and one of the motives that causes problems is that reality does not have a stationary character.

It was suggested by Wang (1977) that in conditions of non-stationary pulse seismic, the recorded trace could be divided into windows where each window would be approximately stationary. Next, a Wiener filter determined specifically for the window in question would be applied. The total filtered trace would be a result of the combination of the individual filtrations in each window (Montenegro and Porsani, 1995). 
When we use a sliding window, with fixed length, the process is called adaptive. The window moves and calculates the filter for each portion of the trace. In this case, for each window position, Levinson's recursion is applied directly to the seismic trace in the data inside the window. The window is shifted by gaining a new sample ahead and losing a sample in the back so as to continue with a fixed number of samples. A new recursion is applied to the trace inside the window. In this way each sample of the seismic trace is deconvolved several times, while the movable window moves. The filtered trace is obtained by calculating the mean value of the number of times each sample was deconvolved.

\section{Surface Related Mutiple Elimination - SRME}

The SRME is a method of filtering that is mainly related to the attenuation of multiple free surface. The great advantage of using this filtering method is the need for only surface knowledge, such as surface reflectivity and source and receiver characteristics.

According to Verschuur (2006) the multiple of first order can be considered to consist of two primary paths, which are connected at the reflection surface point. According to Snell's law at the point of reflection, we can state that the angle of incidence of arrival of the first primary to the receiver must be the same angle of emission of the second primary. Thus, we can combine primary reflections to construct multiple of first order.

Anstey (1966) implemented this method from multiple modeling by the seismic trace autoconvolution, primarily using the idea of trace autocorrelation. Anstey, in 1966, states that the autocorelation function has information about the multiples in a trace.

Considering a seismic response of a ground impulse, without the effect of the surface, is defined by $x_{o}(t)$ and contains all the primary and multiple internal reflections (Verschuur, 2006). If all these events reach the free surface, they will all reflect back. Thus, each event of the primary response will act as a new source. In this way a first order multiple sequence is obtained, convolving the impulsive response itself (Verschuur, 2006):

$$
m_{1}(t)=-x_{o}(t) * x_{o}(t)
$$

the negative sign indicates an inversion of polarity due to surface reflection. If each multiple of first order acts as a new source upon reaching the surface again, the multiple of second order will be written as:

$$
m_{2}(t)=-x_{o}(t) * m_{1}(t)=x_{o}(t) * x_{o}(t) * x_{o}(t),
$$

so to order $n$ we have:

$$
m_{n}(t)=x_{o}(t) * x_{o}(t) * x_{o}(t) * \ldots * x_{o},
$$

The total response $x(t)$, having all multiple of surface is written as:

$$
x(t)=x_{o}(t)-x_{o}(t) * x_{o}(t)+x_{o} * x_{o}(t) * x_{o}(t)-\ldots
$$

The impulsive response $x(t)$ is detected on the surface, but is also reflected back to the subsurface generating a larger order of multiple (Verschuur, 2006). We say then that the total descending field is equal to the original source, $\delta(t)$, combined with the total reflected response $-x(t)$, and this wave field will reflect in the subsurface through the earth response $x_{o}(t)$. This can be written as:

$$
x(t)=x_{o}(t) *[\delta-x(t)]=x_{o}(t)-x_{o}(t) * x(t) .
$$

Thus, all multiple can be generated by the convolution of the primary response with the total response. In the frequency domain we have:

$$
\begin{gathered}
X(f)=X_{o}(f)-X_{o}^{2}(f)+X_{o}^{3}(f)-X_{o}^{4}(f)+\ldots, \\
X(f)=X_{o}(f)-X_{o}(f) X(f),
\end{gathered}
$$

Isolating $X(f)$ and $X_{o}(f)$ in the equation 12, we have:

$$
\begin{gathered}
X(f)=X_{o}(f)\left[1+X_{o}(f)\right]^{-1}, \\
X_{o}(f)=X(f)[1-X(f)]^{-1} .
\end{gathered}
$$

We then obtain the equation 14 where we have the impulse response without the presence of multiple of the surface from the total response. Expanding the equation we have:

$$
X_{o}(f)=X(f)+X^{2}(f)+X^{3}(f)+X^{4}(f)+\ldots
$$

back in time domain:

$$
x_{o}(t)=x(t)+x(t) * x(t)+x(t) * x(t) * x(t)+\ldots
$$

this equation 16 allows to say that the autoconvolution of the total response provides the impulse response free of multiple.

It is assumed in the equations that the seismic trace is the result of a perfect impulse, which we have already seen that is not true. For the real impulse response obtained to be as close to a perfect impulse as possible, deconvolutions will be necessary. When considering the effect of the source, we have:

$$
\begin{gathered}
p_{o}(t)=x_{o}(t) * s(t), \\
p(t)=x(t) * s(t),
\end{gathered}
$$

the equation 10 becomes:

$$
p(t)=x_{o}(t) *[s(t)-p(t)]=p_{o}(t)-x_{o}(t) * p(t),
$$

defining a prediction error operator $a(t)$, it being equal to:

$$
a(t) * s(t)=-\delta(t),
$$

rewriting the equation 19 , we have:

$$
p(t)=p_{o}(t)+p_{o}(t) * a(t) * p(t) .
$$

The operator $a(t)$ acts as the source deconvolution filter. The effect of surface reflectivity is described by the scale factor -1 . The equation 16 including the effect of the source, is written as:

$p_{o}(t)=p(t)-a(t) * p(t) * p(t)+a(t) * a(t) * p(t) * p(t) * p(t)-\ldots$

Representing the removal of multiples defined by the equation 22, we have:

$$
P_{o}(f)=P(f)-A(f) P^{2}(f)+A^{2}(f) P^{3}(f)-A^{3}(f) P^{4}(f)+\ldots,
$$

where the operator $A(f)$ is defined as:

$$
A(f)=-\left[S(f)^{-1}\right],
$$

$S(f)$ is the direct Fourier temporal transform of the source signature $s(t)$. 


\section{Results}

The results sought to evaluate each deconvolution within its particularity, to improve the quality of the velocity analysis through the removal of multiples, to show that the SRME filtering is associated with the deconvolution of the trace, and finally to associate the best result of the filtration with the best of deconvolution.

\section{Deconvolution}

The spiking deconvolution (Figure 1) was tested for several coefficients, but the best result was obtained when the number of coefficients was equal to 1 .

The spiking deconvolution (Figure 2) performed in the Seisspace was not satisfactory.

The norm $L_{p}$ deconvolution (Figure 3 ) was evaluated by means of the number of filter coefficients, the value of the norm $L_{p}$ and the number of iterations. A norm was used with $p=1$ and the number of iterations equal to 100 .

The adaptive deconvolution (Figure 4) was tested using fixed time windows, and fixed coefficient number. The parameters chosen were number of coefficients equal to 1 and number of channels equal to 1 .

Although all the results developed by FORTRAN.90 have been satisfactory, we can observe that the section that presented the best compression of the seismic pulse is associated with deconvolution using norm $\mathrm{Lp}$.

\section{Influence on Amplitude Spectrum}

Analyzing the spectra of each deconvolution in FORTRAN.90 (Figures $6,7,8$ ) it is possible to notice a highlight in the information contained in the frequency band above $35 \mathrm{~Hz}$ in relation to the original data (Figure 5 ). This result is consistent with the goal of deconvolution that is to try to get back the reflectivity function. The deconvolution amplitude spectrum realized in PROMAX (Figure 9) has a very anomalous frequency band as compared to the original.

\section{Surface Related Multiple Elimination - SRME}

In order to test the different methods, we separated the CMP 2965 from the line 214-RL-0266 of the Jequitinhonha Basin, because this CMP presents the well-defined free surface multiples as well as some reflections. The figures: $15 ; 16 ; 17 ; 18$, show the comparison between the original CMP and the CMP after the application of each of the methods.

\section{Influence of filtrations on the velocity spectrum}

The sections obtained from the SRME filtering method associated with deconvolutions were generated from the second velocity analysis, using as input data for this analysis the filtered SRME data. Initially the original data or raw data was processed, without any filtering method being applied, this served as a comparison effect of the effects and improvements after the application of the SRME associated the deconvolutions. Among the deconvolutions performed in FORTRAN.90, the one that presented the best results for SRME application was the deconvolution using norm $L_{p}$, however, it is only possible to observe differences between these deconvolutions through a high degree of detail.

\section{Conclusion}

The attenuation of the surface multiples in line 2140266 of the Jequitinhonha Basin using the SRME filtering method associated with the deconvolutions was satisfactory. The deconvolutions used served as input data for SRME application, because according to the theory of this method, for the prediction of the surface multiple, the pulse must be considered unitary. In this way, given the SRME prerequisite, the data was better attenuated.

Due to the geological complexity of the site there are complications to attenuate the surface multiple of the shallow platform region. However, the combination of the two methods (deconvolution and SRME) proved to be efficient for the attenuation of the deep water surface multiple.

\section{Acknowledgements}

This research was supported by CNPq and INCTGP/CNPq. The facility support from CPGG/UFBA is also acknowledged.

\section{References}

Akaike, H., 1969, Fitting autoregressive models for prediction: Annals of the institute of Statistical Mathematics, 21, 243-247.

Anstey, N. A., 1966, The sectional auto-correlogram and the sectional retro-correlogram: Geophysical Prospecting, 14, 389-426.

Ibrahim, Z., 2014, AnÃ $i l i s e ~ t e m p o-f r e q u \tilde{A}^{a}$ ncia do sinal sÃsmico utilizando a distribui $\tilde{A} \S \tilde{A} \$ o$ wigner-ville e o mÃ $\left(\right.$ todo de $m \tilde{A}_{j} x i m a$ entropia: Aplicada para a estimativa do fator $q$ e de atributos.

Lima, R. R. and M. J. Porsani, 2013, Atenuação do groundroll utilizando filtragem adaptativa svd no doíinio da frequência: 13th International Congress of the Brazilian Geophysical Society \& EXPOGEF, Rio de Janeiro, Brazil, 26-29 August 2013, 1488-1493.

Melo, P. E. M. and M. J. Porsani, 2001, Deconvolução iterativa de dados sísmicos com norma Lp: Presented at the 7th International Congress of the Brazilian Geophysical Society, SBGf, Salvador.

Montenegro, J. B. and M. Porsani, 1995, Deconvoluçăo adaptativa da assinatura da fonte utilizando um algoritmo tipo Levinson para janelas de tempo: Presented at the 4th International Congress of the Brazilian Geophysical Society, SBGf, Rio de Janeiro.

Popini, M. V., M. J. Porsani, and J. L. Porsani, 2001, Processamento de dados gpr utilizando métodos da sísmica de reflexão: Presented at the 7th International Congress of the Brazilian Geophysical Society, SBGf, Salvador.

Porsani, M. J., S. Niwas, and N. R. Ferreira, 2001, Robust inversion of vertical electrical sounding data using a multiple reweighted least-squares method: Geophysical prospecting, 49, 255-264.

Verschuur, D. J., 2006, Seismic multiple removal techniques: Past, present and future: EAGE Publications, Netherlands.

Wang, R., 1977, Adaptive predictive deconvolution of seismic data: Geophysical Prospecting, 25, 342-381.

Yilmaz, O., 1987, Seismic data processing Society of Exploration Geophysicists Tulsa: OK Google Scholar. 


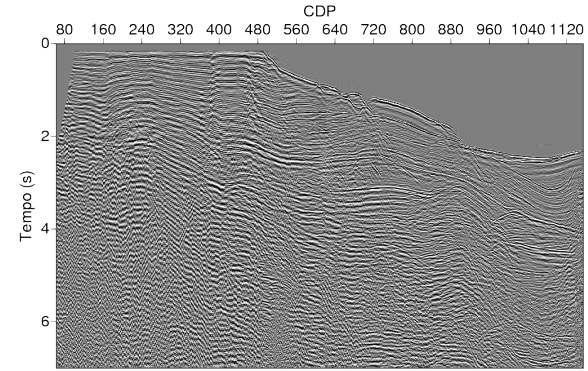

Figure 1: Section stacked using the final velocity, resulting from the spiking deconvolution applied by the FORTRAN language.

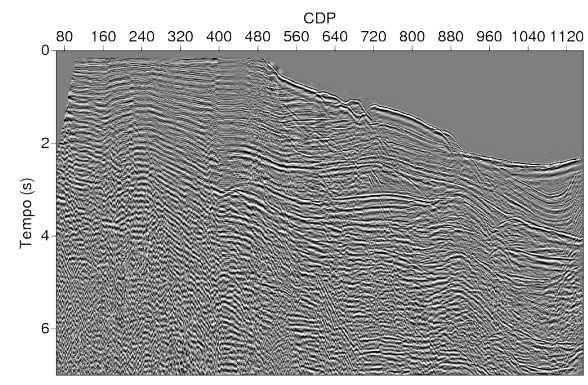

Figure 2: Section stacked using the final velocity, resulting from the spiking deconvolution applied by Seisspace software.

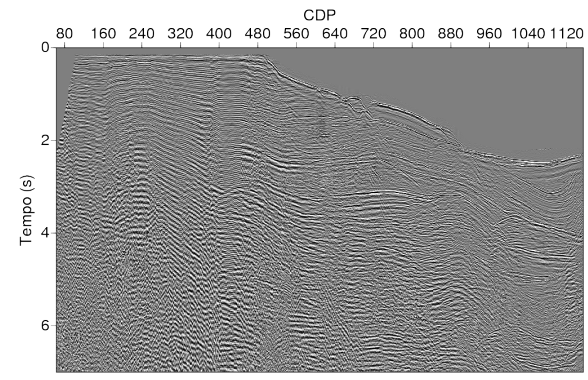

Figure 3: Section stacked using the final velocity, resulting from the deconvolution with norm $L_{p}$.

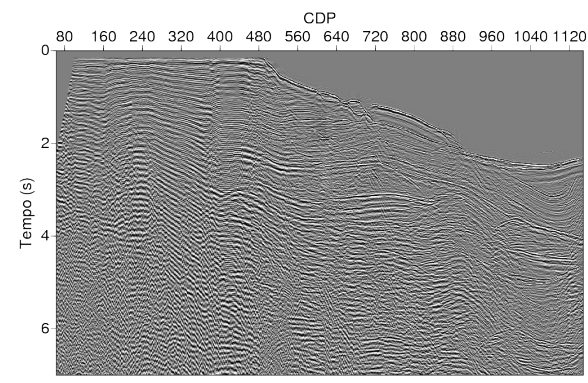

Figure 4: Section stacked using the final velocity, resulting from the adaptive deconvolution.

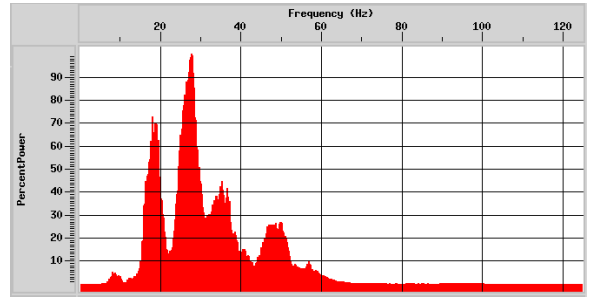

Figure 5: Amplitude spectrum of the raw data.

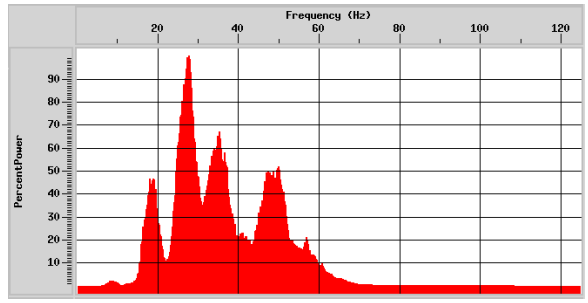

Figure 6: Amplitude spectrum of spike deconvolution developed in FORTRAN.

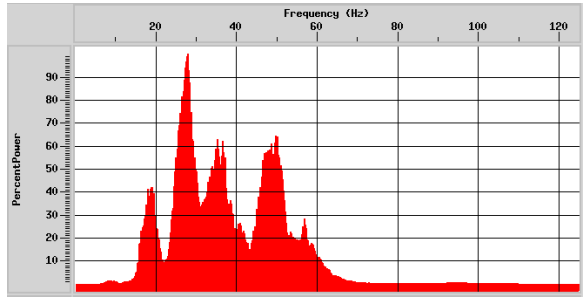

Figure 7: Amplitude spectrum of the adaptive deconvolution developed in FORTRAN.

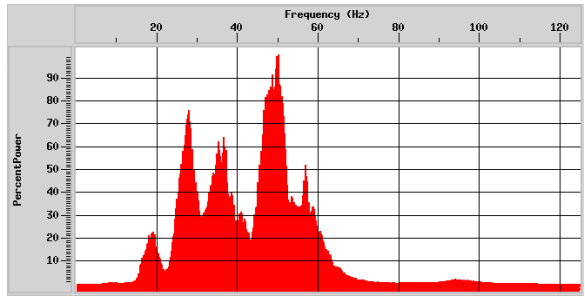

Figure 8: Amplitude spectrum of the deconvolution with norm $L_{p}$ developed in FORTRAN.

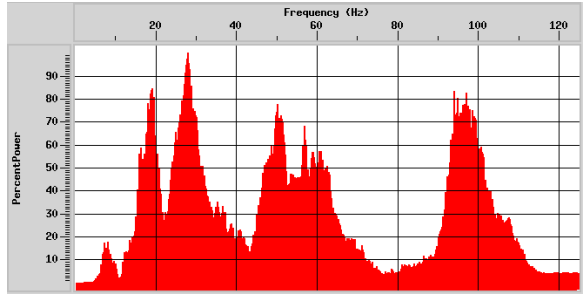

Figure 9: Amplitude spectrum of spike deconvolution developed in Seisspace.. 


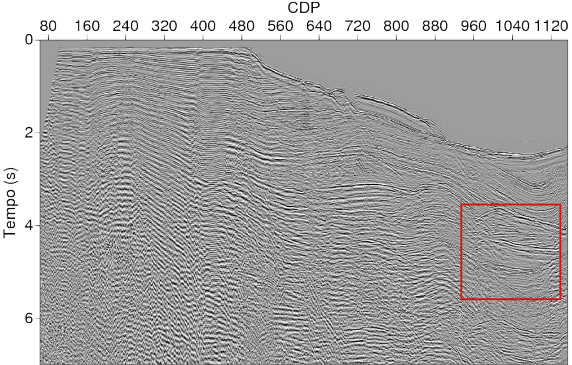

Figure 10: Region of the stacked seismic section used to perform the comparison between deconvolutions and SRME filtering.

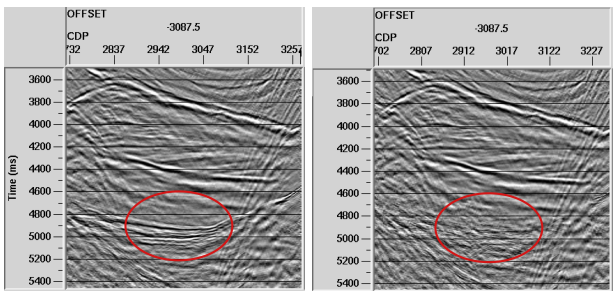

Figure 11: Result of the SRME method (right) after Seisspace deconvolution (left).

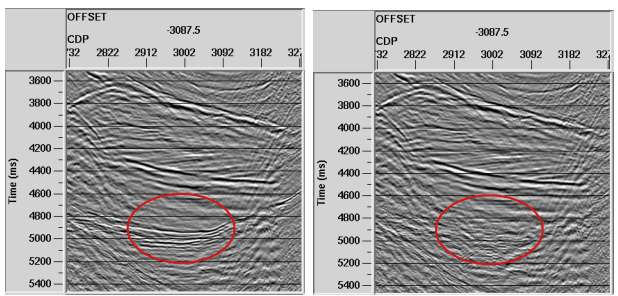

Figure 12: Result of the SRME method (right) after the spiking deconvolution in FORTRAN (left).

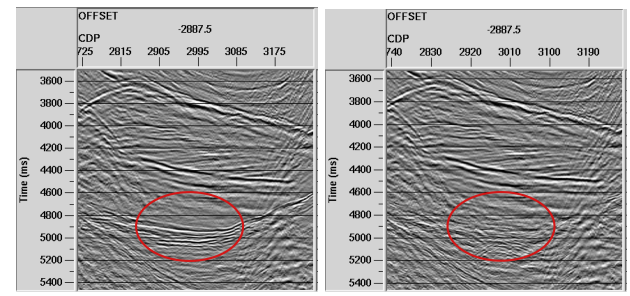

Figure 13: Result of the SRME method (right) after the adaptive deconvolution (left).

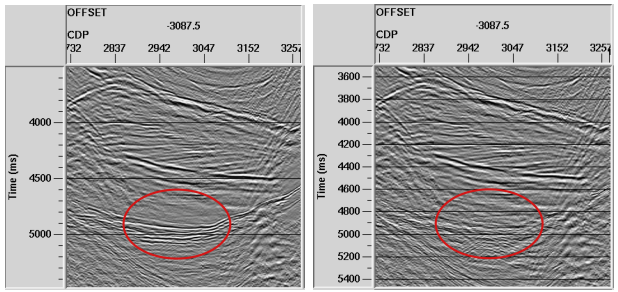

Figure 14: Result of SRME method (right) after deconvolution with norm $L_{p}$ (left).

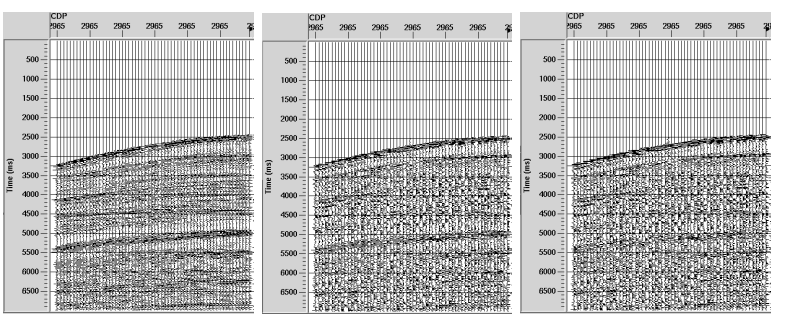

Figure 15: Result of the SRME method (right) using the Seisspace spiking deconvolution (center).

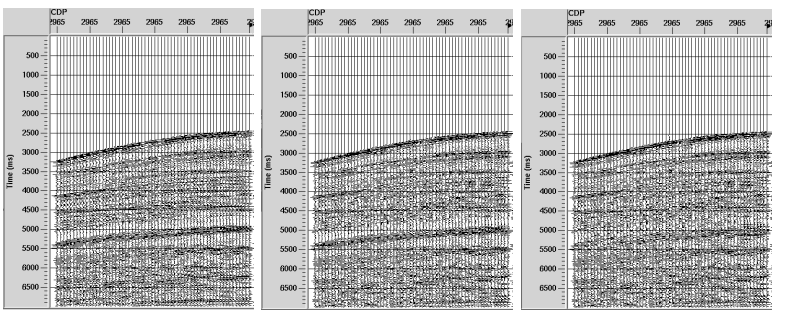

Figure 16: Result of the SRME method (right) using the spiking deconvolution in FOTRAN (center).
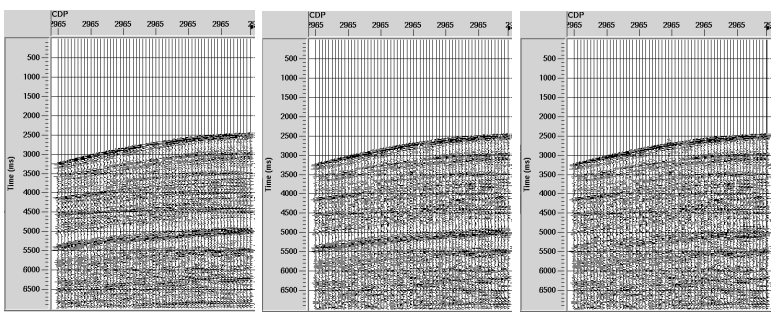

Figure 17: Result of the SRME method (right) using the adaptive deconvolution (center).
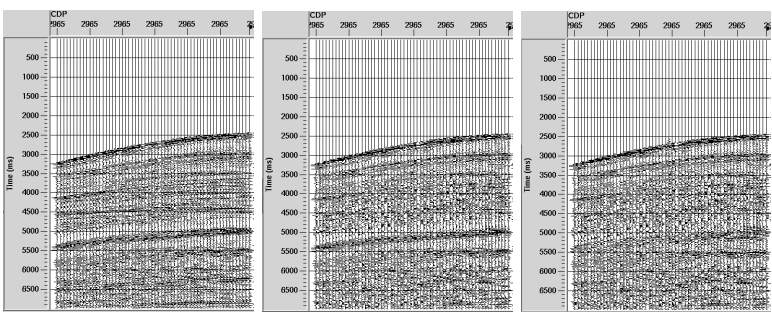

Figure 18: Result of SRME method (right) using deconvolution with norm $L_{p}$ (center). 